\title{
Characterization and functions of Nano-Wool crystallites
}

\author{
Bin $\mathrm{Wu}^{1, \mathrm{a}}$, Yan $\mathrm{Yi}^{1, \mathrm{a}}$, Tao $\mathrm{Xu^{1, \textrm {b } }}$, Hua Lei ${ }^{1, \mathrm{c}}$, \\ ${ }^{1}$ The Materials and Metallurgy College, Guizhou University, \\ Guiyang, Guizhou, P.R.China, 550003 \\ axxlhtt@sohu.com, bxxlhtt@sina.com, ${ }^{\mathrm{c} x x I h t t @ o v i . c o m}$
}

Keywords: Nano-wool; Protein; Characteristic, Functions, Wash durability

\begin{abstract}
In the research, we reported on a new method to produce fine wool protein crystallites by regeneration method, and this kind protein emulsion can be used on the treatment of fabrics, synthetic or nature fibers, allowing to form protein sheath or encapsulation layer that do not disturb the original properties and adding additional functions. From the structure analysis, it was showed that the particle size of the protein is below 100nm and micro-morphology being ball-like in appearance. There exists mixed crystalline morphology, single- and multi-crystal , in the nano-wool protein crystallites and it also showed higher crystalline degree (crystallinity) than original wool materials. And, cotton woven fabric treated with this kind nano materials by simply method, exhibits several unique features and after standard washing, these functions still remained .
\end{abstract}

\section{Introduction}

Natural fibers, Silk and wool, have played an important role in textile material from ancient time and still have been widely used in the modern textiles industry for their unique properties as high quality textile material. However, for the limitation/requirement in the stage of spinning, not all fibers can be used to spin, because of their short length. Therefore, sometimes there are some natural fibers such like wool, silk, cotton or hemp etc. is wasted during processing. To develop a new way to reuse those fibers has large marketing potential because of their excellent intrinsic properties. Meanwhile, not only textile industry, many other industries also need such technology in new material or functional design or application.

So many researchers, especially in Japan, try to develop new applications for those fibers because their excellent intrinsic properties can be used to develop new materials in biotechnological and biomedical fields. In further study, moderate quantities of silks and silk-mimic biopolymers have been achieved due to advances in molecular biotechnology and protein engineering. However, $100 \%$ regenerated silk fiber has not been industrially produced because no proper spinning technology has come out[1,2].

However, material properties depend not only on the macromolecule but also on the structure the way the macromolecule is put together[2-6]. Silk fibroin powder has already found its utility as cosmetic materials and functional foods. Powdered silk fibroin is considered effective as an additive for cosmetic and pharmaceutical preparations because of its moderate moisture absorption and retention properties, and its high affinity for human skin and silk; actually, wool powder also preserve these virtues. Silk powder is one of the useful physical forms of silk fibroin protein, as well as fiber and film, for biomaterial applications[7,8].

Usually, compared with inorganic fiber, polymer fibers not only have high strength but also high break intensity and high elongation. So they are very difficult to crush into a small powder. Moreover, even they are cut into short pieces, they still tend to wind together to form balls during grinding. In order to get fine silk powder, usually wool and silk fibroin powders are produced by special chemical pretreatment to destroy the chemical bond and to reduce the crystallinity[2,9,10].

As for wool fiber, it is cheaper and friendly to the human skin; its strength is less than silk fiber. Due to their low price and availability, these films are more necessary to be developed into fine powder, especially for those unsuitable for the spinning of yarn, as they are small in diameter and their performance are very excellent than the longer and stronger fibers that are more welcome in 
the textile industry. As is well known, keratin fibers, such as those found in wool, feel rich due to their excellent hygroscopic and moisture releasing properties, which respond to the moisture content of the air. Such properties are characteristic of a complex composed of a fibrous keratin with .alpha.-helix structure, which is the crystalline moiety of the fiber, and an amorphous matrix protein, the molecules of which are cross-linked with each other through disulfide bonds (--SS--). Because of less research on the wool superfine functional powder compared with silk, so, in our research, we have developed a new method to produce fine wool protein crystallites by regeneration method, and this kind protein emulsion can be used on the treatment of fabrics, synthetic or nature fibers, allowing to form protein sheath or encapsulation layer that do not disturb the original properties and adding additional functions. We report in this article on some results on the powder structure properties and its functions.

\section{Material and methods}

The solution was prepared by alkaline catalyzed hydrolysis of nature wool fibers as wool fibers immersed in the alkaline environment by optimized hydrolysis temperature and time. The latter is the process of an nano-emulsification method formed order crystal phase emulsion comprising spherical particle of protein crystallites in nano-scale with electrolyte solution that was a kind of regeneration method.

Particle size distributions of the protein emulsion, which was tested on a LS 13320 Particle Size Analyzer.

The molecule chain structure were analysed by FTIR, KBr disc technique is the method used in the preparation of nano-wool protein crystallites for infrared analysis to judge if any structure variations.

The phase structure of the produced nano-wool powders was identified by X-ray diffraction (XRD)with CuK incident radiation at $40 \mathrm{kV}$. The morphological and the compositional characterizations of the nano-materials were performed by using a TEM (PHILIPS CM120) equipped with EDS(EXL).

Cotton fabrics were treated by nano wool emulsion with dip-pad-dry-curing method. The functional tests of the fabrics washed and un-washed were done.

\section{Results and discussion}

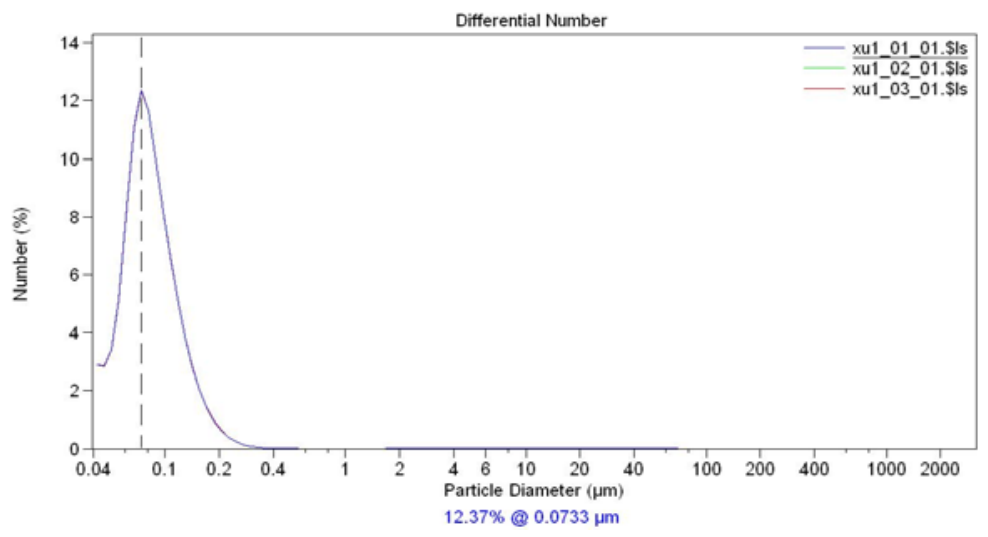

Fig.1. Result of particle analysis

Protein particle size analysis. The particle size distributions of the protein emulsion are analyzed by using LS 13320 Particle Size Analyzer. The results are shown in Fig.1. The resulted showed the particle size below 100nm (about $70 \mathrm{~nm}$ ) under certain condition of preparation technology.

Morphology of Crystallites. From TEM analysis, Fig.2, showed the image of wool protein crystallites. The individual crystallites, prepared by hydrolysis-regeneration method, were ball-like in appearance. 


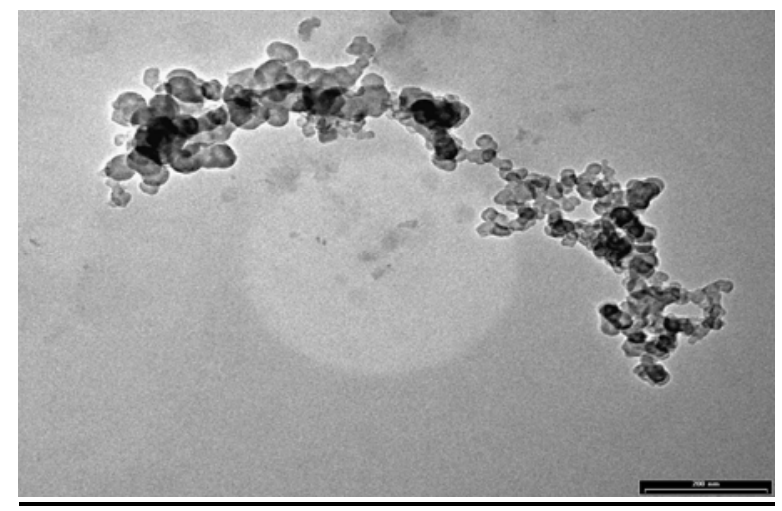

Fig.2. TEM micrograph of wool protein crystallites prepared by hydrolysis method

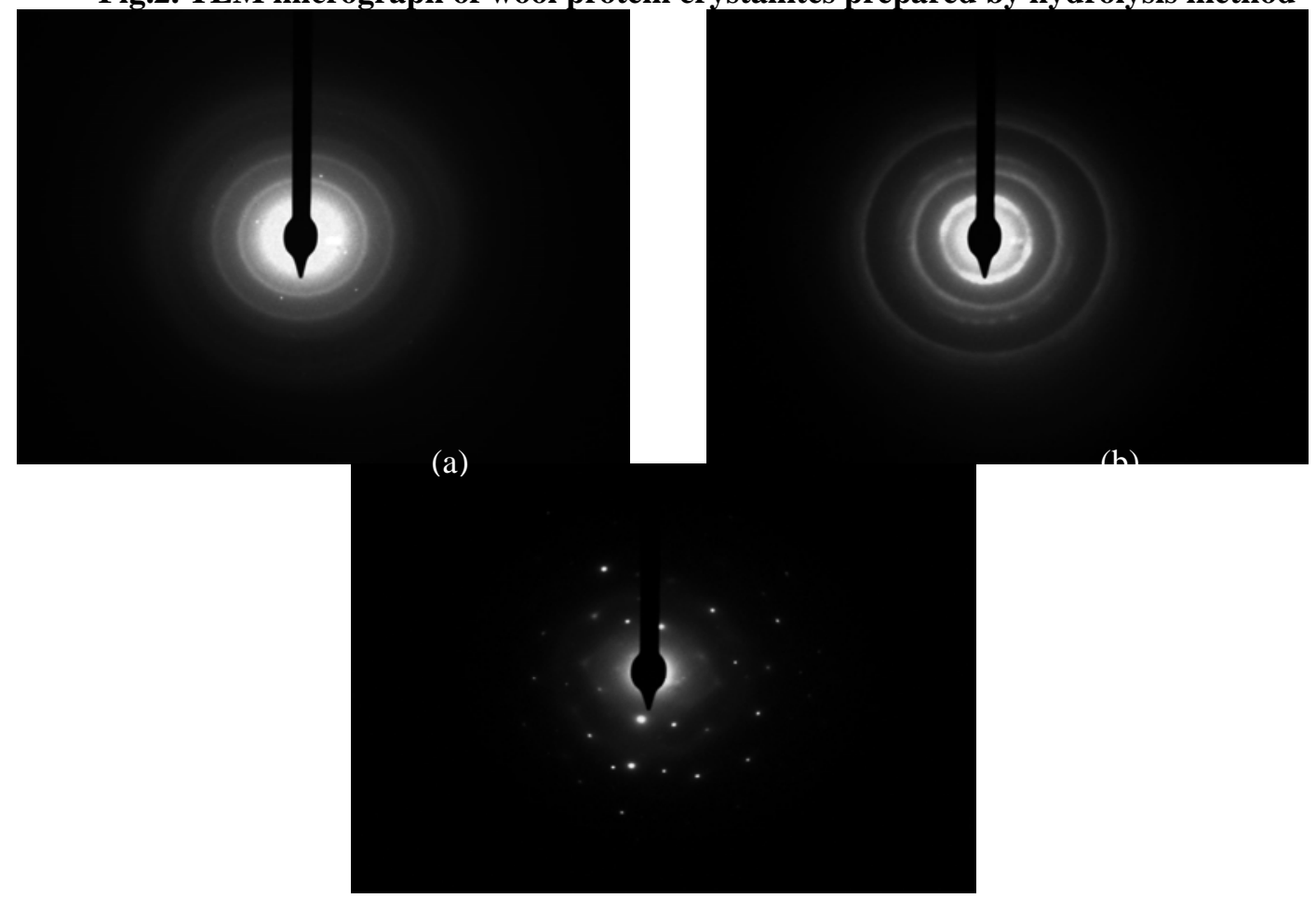

Fig.3. TEM observation for diffraction pattern ring of nano-woo protein crystallites

Protein lattice diffractive ring[Fig3.(a),(b)]; protein single crystal structure diffractive spot [Fig3.(c)]were observed. And, from the result of Fig.3(b), the information of overlap structure of protein multi-crystal and single-crystal was also observed. To resolve the crystalline structure of this kind of protein crystallites, more deep fine experiments are needed.

FTIR analysis for nano-wool protein.

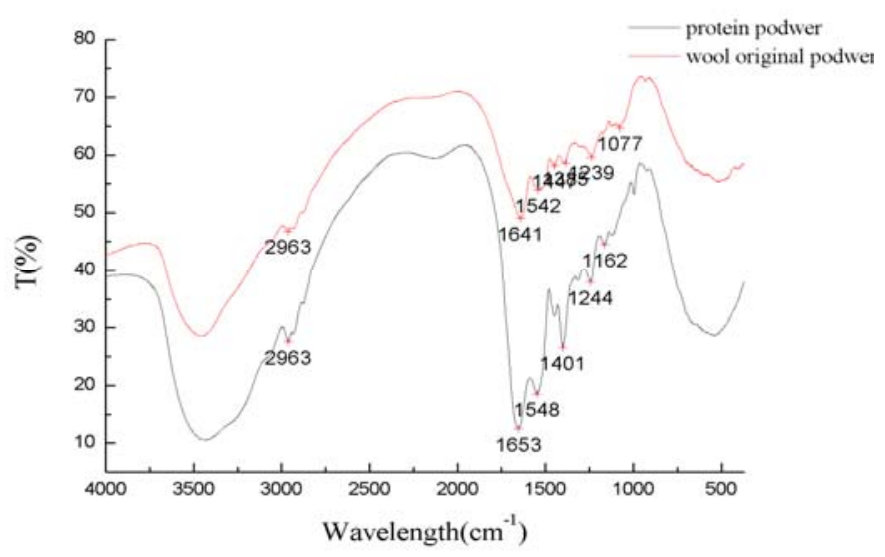

Fig.4. FTIR analysis curves

From the results of Fig.4., Several absorption peak appeared, $1653 \mathrm{~cm}^{-1}$ absorption peak showed 
stretching vibration of $\mathrm{C}=\mathrm{O}$ bond of polypeptide linkage; $1548 \mathrm{~cm}^{-1}$ showed the trans-structure of peptide chain and $1401 \mathrm{~cm}^{-1}$ showed cis-structure of peptide chain. Nano-wool protein crystallites have not any structure differences with wool original powder. And these functional bond could be used for combining the nano wool materials to cotton fabric.

XRD analysis for nano-wool protein.

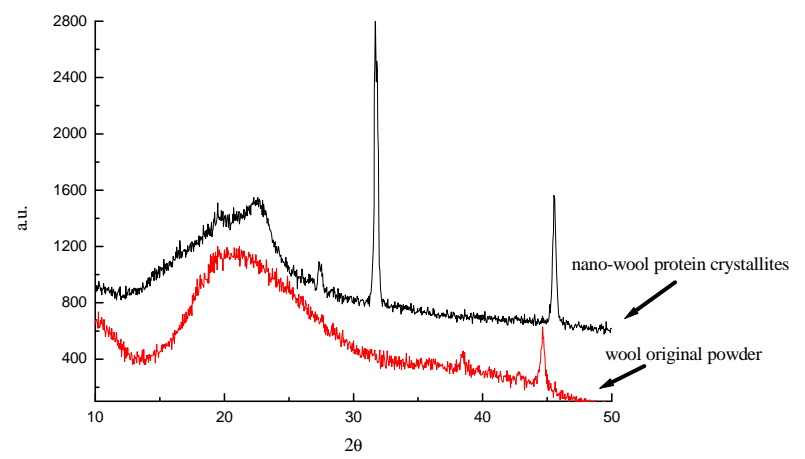

Fig.5.XRD curves of powder

$\mathrm{XRD}$ analysis for the samples above, it was showed that ,when wool protein regeneration after hydrolysis, there have higher crystalline degree (crystallinity)for nano-wool protein crystallites under the preparation technology. From the result of Fig.5, sharp diffraction peak appeared.

From the experiments above, wool protein within nano scale could be made by special technology. Ball-like ordered structure can form and showed higher crystallinity than original wool material. This kind of protein crystallites include also both multi- and single crystal structure, it is maybe a kind of overlap structure composites and have not any chain structure differences with original wool. The type of materials can be treated on fabric, synthetical or nature. The further research results will be reported latter.

\section{Conclusion}

As for wool fiber, it is cheaper and friendly to the human skin; we developed a new method to produce fine wool protein crystallites by regeneration method, and this kind protein emulsion can be used on the treatment of fabrics, synthetic or nature fibers, allowing to form protein sheath or encapsulation layer that do not disturb the original properties and adding additional functions.

\section{Acknowledgement}

The authors sincerely acknowledge the financial support for the project by the Science Foundation of Guizhou Province (Serial number: 2003JN027) and the Doctoral Foundation of Guizhou University (Serial number:2007-008) and SRT project of GZU(Innovation training 22)

\section{References}

[1] Yao J, Masuda H, Zhao C, Asakura T. Macromolecules 2002,35 (1), 6 - 9.

[2] Tsubouchi K. U.S. Pat5,853,764, December 29, 1998

[3] Lu X, Akiyama D, Hirabayashi K. J Sericultural Sci Jpn 1994,63(1), 21-27.

[4] Takeshita T, Ishida K, Kamiishi Y. Macromol Mater Eng 2000,283, 126-131.

[5] Xu T, Yu J, Jin Z H. Mater Design 2001,22, 27-31

[6] Xu T, Lei H, Xie C S. Polym Test, 2002,21,319-324

[7] Freddi G, Tsukada M, Beretta S. J Appl Polym Sci 1999, 71, 1563-1571.

[8] Tanaka T, Tanigami T, Yamaura K. Polym Int 1998, 45(2),175-184.

[9] Yamada M, Narita S, Kondo T, et al. U.S. Pat5,276,138, January 4, 1994

[10] Arai K, Nojiri H, Naito S. U.S. Pat5,763,583, June 9, 1998 